The Astrophysical Journal, 394:662-669, 1992 August 1

(C) 1992. The American Astronomical Society. All rights reserved. Printed in U.S.A.

\title{
DEVELOPMENT OF MAGNETOHYDRODYNAMIC TURBULENCE IN CORONAL LOOPS
}

\author{
DANIEL O. GÓMEZ ${ }^{1}$ \\ Instituto de Astronomía y Física del Espacio, CONICET, c.c. 67, Suc. 28, (1428) Buenos Aires, Argentina \\ AND \\ CONSTANTINO Ferro Fontán \\ Department of Physics, Facultad de Ciencias Exactas y Naturales, University of Buenos Aires, Ciudad Universitaria, (1428) \\ Buenos Aires, Argentina \\ Received 1991 May 28; accepted 1992 February 10
}

\begin{abstract}
We propose that a stationary spectrum of magnetohydrodynamic turbulence is generated in solar coronal loops by the interweaving of magnetic field lines driven by the turbulent velocity field in the convective region. Starting from the set of incompressible magnetohydrodynamic equations, we derive two ordinary differential equations describing the stationary turbulent energy spectrum and the frequency of direct energy cascade for a given kinetic energy spectrum of the subphotospheric convective motions.

We obtain an estimate of the typical dissipation length scale, at which magnetic energy efficiently heats the plasma by Joule dissipation. The impact of recent results of two-dimensional magnetohydrodynamic numerical simulations on the coronal heating problem is also discussed.
\end{abstract}

Subject headings: MHD - Sun: corona - turbulence

\section{INTRODUCTION}

The highly resolved X-ray observations of the solar corona obtained during the last two decades, systematically indicate the presence of quite inhomogeneous magnetic structures (Vaiana \& Rosner 1978). The very active role of the magnetic energy contained in these structures in heating the corona, has since been intensively studied (see Kuperus, Ionson, \& Spicer 1981 for a review).

The subphotospheric fluid motions permanently stress the magnetic field lines that constitute the above mentioned structures, also known as coronal loops. In this way, the convective region replenishes the magnetic energy which is continually being dissipated. The subphotospheric velocity field provides enough energy input to compensate for the conductive and radiative losses of the coronal plasma. However, the problem of coronal heating is far from being solved, since the Joule relaxation of the above mentioned magnetic stresses is very slow. Parker (1981a, b, 1983a, b) suggests that footpoint motions of cor onal loops continuously drive the magnetic field into states of dynamical nonequilibrium in which current sheets spontaneously form. Parker asserts that the magnetic reconnection taking place in this stochastic distribution of small current sheets, both enhances the ohmic dissipation of magnetic energy and simplifies the stressed magnetic structures (topological dissipation).

Hollweg (1983) introduced the idea of excitation and dissipation of turbulence as an alternative way of efficiently heating the solar corona. Van Ballegooijen (1986) presents a model of the initial stages of excitation of MHD turbulence. He calculates the evolution of a force-free field driven by the turbulent subphotospheric velocity field and demonstrates the development of a direct energy cascade to the large wavenumbers spectral region. A more detailed overview on different mechanisms of coronal heating can be found in Narain \& Ulmschneider (1990; also Gómez 1990).

\footnotetext{
${ }^{1}$ Presently at Institute for Astronomy, University of Hawaii.
}

It is well known that when a three-dimensional incompressible fluid is submitted to external forcing with a narrow spectrum, a direct energy cascade is generated and a stationary Kolmogorov energy spectrum is achieved. This is also the case in two- and three-dimensional MHD turbulence. In coronal loops, the mean magnetic field (oriented along the axis of the loop) is large compared to the turbulent fluctuations of the magnetic and velocity fields, thus making the flow to be highly anisotropic. While the perpendicular motions may develop small dissipative scales giving rise to turbulent dissipation, spatial variations along the mean field generally remain smooth, their dynamics being determined by weak interaction of Alfvén waves (Biskamp \& Welter 1989). In the present work we are concerned with the two-dimensional dynamics of velocity and magnetic fluctuations in planes perpendicular to the mean magnetic field, which is externally driven by the stochastic subphotospheric motions.

According to the cascade theories (see Hasegawa 1985), an energy intertial range in $k$ space is formed, bridging the gap between the low-wavenumber energy containing region (granules wavenumber in the coronal case) and the largewavenumber dissipative structures (current sheets). From simple scaling considerations, a $k^{-5 / 3}$ energy spectrum can be derived not only for three-dimensional hydrodynamic systems (Kolmogorov 1941) but also for the two- and threedimensional MHD cases. For MHD systems, a modification to Kolmogorov's argument (leading to a $k^{-3 / 2}$ energy spectrum) has been suggested, based on the ability of Alfvén waves to transfer energy in regions of large-scale magnetic fields (Kraichnan 1965). The existence of a broad-band inertial range has repeatedly been confirmed in numerical simulations (Fyfe, Joyce, \& Montgomery 1977; Fyfe, Montgomery, \& Joyce 1977; Matthaeus \& Lamkin 1986). However, some uncertainties still remain with respect to the exact exponent $(-3 / 2$ or $-5 / 3)$ of the power-law energy spectrum in this region (Biskamp \& Welter 1989; Politano, Pouquet, \& Sulem 1989).

Gómez \& Ferro Fontán (1988) showed that Joule dissipation of stationary MHD turbulence is a very plausible 
mechanism for coronal heating. They suppose that the subphotospheric forcing has a narrow spectrum whose wavelength is around the length scale of granular convection $\left(10^{3} \mathrm{~km}\right)$. Unfortunately, we are still unable to observationally test these theoretical predictions. Typical length scales for structures associated with the energy cascade region $\left(\ll 10^{3} \mathrm{~km}\right)$ are still beyond the present-day capabilities of spatial resolution. The range of length scales that we are able to resolve lies mostly in the energy-containing region, where the terms describing the interaction between the coronal magnetic field and the photospheric motions are as important as the nonlinear terms responsible for the cascading mechanism. Also, there is no observational evidence in support of a narrow spectrum for the subphotospheric stressing mechanism in this length scale range. Quite the contrary, recent observational studies based on Doppler imaging of photospheric motions (Zahn 1987; Chou et al. 1991; Tarbell 1992), strongly indicate the existence of a broadband, power-law kinetic energy spectrum, as expected for a turbulent medium like the subphotospheric convective region. The aim of the present paper is to present a simplified theoretical model for coronal MHD turbulence, which however is able to make theoretical predictions of observable quantities in the energy containing spectral region.

The energy spectrum in the energy containing region (which is also the large-scale region) does not display the universal features of the spectrum in the inertial range, being strongly dependent on the characteristics of the external driving force. A phenomenological model has been developed (Canuto \& Goldman 1985) which describes bulk properties of the largescale turbulent spectrum. This approach extends Heisemberg's theory of isotropic turbulence (Heisemberg 1948; also Batchelor 1953, pp. 125-168) to the energy containing region. It has already been applied to other problems of astrophysical interest, like convective regions in stellar interiors (Canuto \& Goldman 1985; Canuto, Goldman, \& Chasnov 1987), velocity versus size relationship in molecular clouds (Canuto et al. 1985 ) or turbulent viscosity in accretion disks (Canuto, Goldman, \& Hubickyj 1984). For three-dimensional incompressible fluids, Canuto et al. (1987) derive a set of ordinary differential equations (coupled to an integral condition) for both the energy spectrum and eddy correlation rate.

In the present paper we extend the analysis of Canuto et al. (1987) to two-dimensional MHD turbulence and calculate the turbulent magnetic energy spectrum of coronal loops for a given Fourier distribution of the subphotospheric velocity field. In $\S 2$ we derive the equation for the turbulent energy spectral function. In $\S 3$ we briefly describe the heuristic model of Canuto et al. (1987) and apply it to MHD turbulence in the solar corona. In $\S 4$ we describe the numerical solution for the model equations and show the results. In $\S 5$ we discuss our results in the context of the coronal heating problem.

\section{EQUATION FOR THE TURBULENT MAGNETIC ENERGY SPECTRUM}

We consider a coronal loop of length $L$ with a cross-section $D \times D$. We discard toroidal effects because $D \ll L$. The overall magnetic field is parallel to the axis of the loop as depicted in Figure 1. The planes $z=0$ and $z=L$ correspond to the solar photosphere. We assume the beta of the plasma (ratio of gas pressure to magnetic pressure) to be much smaller than one in the coronal part of the loop, leaping discontinuously to a value larger than one at the photospheric plates. The assumption (supported by the steep increase of mass density with depth at

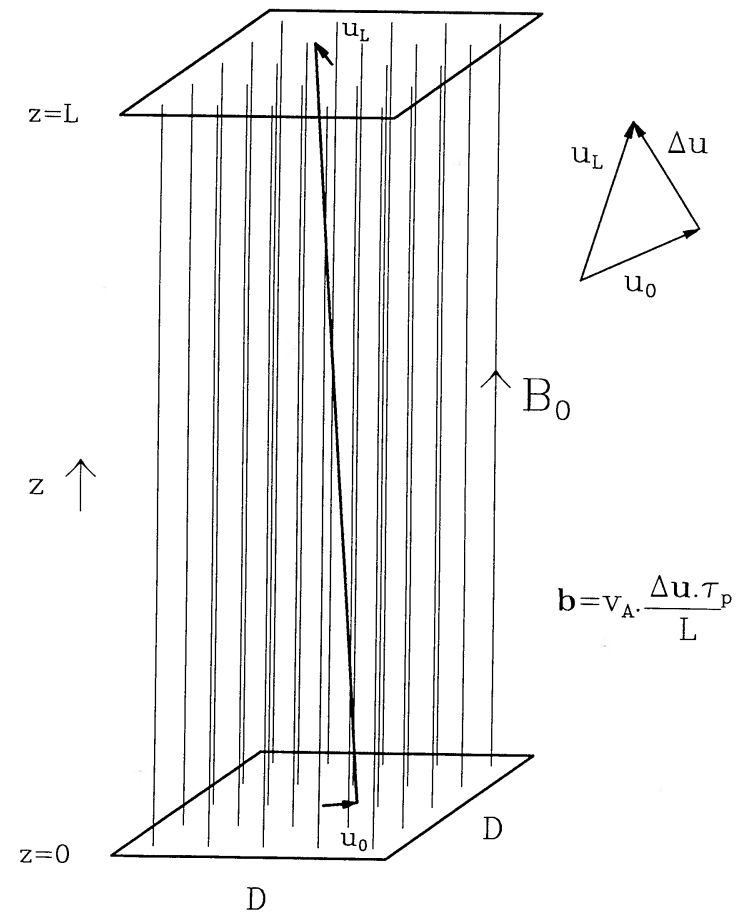

Fig. 1.-Perturbation of a single field line of a coronal loop. The planes $z=0$ and $z=L$ represent the photosphere at each footpoint. The vector diagram (right) shows the magnetic component $\boldsymbol{b}$, which is parallel and proportional to $\Delta u=u_{L}-u_{0}$.

the photosphere), combined with the very high electric conductivity of the plasma, implies that the photospheric convective motions will easily drive magnetic stresses in the corona.

As time goes by, the stochastic subphotospheric velocity field generates fluctuating velocity and magnetic field components in the corona, both in planes perpendicular to the main $\boldsymbol{B}_{0}$ field. Expressing both fields in velocity units, we have

$$
\begin{gathered}
\boldsymbol{B}=v_{\mathrm{A}} z+\boldsymbol{b}(x, y, z, t), \quad \boldsymbol{b} \cdot \boldsymbol{z}=0, \\
\boldsymbol{u}=\boldsymbol{u}(x, y, z, t), \quad \boldsymbol{u} \cdot \boldsymbol{z}=0,
\end{gathered}
$$

where $v_{\mathrm{A}}=B_{0}(4 \pi \rho)^{-1 / 2}$ is the Alfvén velocity of the plasma ( $\rho$ : mass density). The evolution of the Fourier components $u_{k}(z, t)$ and $\boldsymbol{b}_{\boldsymbol{k}}(z, t)$ is governed by the following MHD equations:

$$
\begin{aligned}
\left(\partial_{t}+\eta k^{2}\right) \boldsymbol{b}_{k} & =v_{\mathrm{A}} \partial_{z} \boldsymbol{u}_{\boldsymbol{k}}+i \sum_{\boldsymbol{k}=\boldsymbol{k}_{1}+\boldsymbol{k}_{2}} \boldsymbol{k} \times\left(\boldsymbol{u}_{\boldsymbol{k}_{1}} \times \boldsymbol{b}_{\boldsymbol{k}_{2}}\right), \\
\left(\partial_{t}+v_{1} k^{2}\right) \boldsymbol{u}_{k}= & v_{\mathrm{A}} \partial_{z} \boldsymbol{b}_{k}+i \sum_{\boldsymbol{k}=\boldsymbol{k}_{1}+\boldsymbol{k}_{2}} \\
& \times\left[\left(\boldsymbol{k}_{1} \times \boldsymbol{b}_{\boldsymbol{k}_{1}}\right) \times \boldsymbol{b}_{\boldsymbol{k}_{2}}-\left(\boldsymbol{k}_{2} \cdot \boldsymbol{u}_{\boldsymbol{k}_{1}}\right) \boldsymbol{u}_{\boldsymbol{k}_{2}}\right]_{\perp}, \\
\boldsymbol{k} \cdot \boldsymbol{u}_{k}= & 0=\boldsymbol{k} \cdot \boldsymbol{b}_{\boldsymbol{k}},
\end{aligned}
$$

where

$$
\left[A_{i}\right]_{\perp}=A_{i}-k^{-2} k_{i} k_{j} A_{j}
$$

is the component of $\boldsymbol{A}$ perpendicular to $\boldsymbol{k}=\left(k_{x}, k_{y}, 0\right)$. The coefficients $\eta$ and $v_{1}$ correspond to Joule and viscous dissipation, respectively. Note that the viscosity coefficient $v_{1}$ is much smaller than the Newton viscosity $v_{0}\left(v_{0} \approx p \tau_{i}, p\right.$ : pressure, $\tau_{i}$ : ion collision time), due to the effect of a strong magnetic field on the transport properties of the plasma (Braginskii 1965). 
The terms $v_{\mathrm{A}} \partial_{z}$ represent the coupling of turbulence in a plane $z=z_{0}$ with nearby planes. The role of these terms is crucial for the development of turbulence, since it is through them that fluctuations of the fields can be pumped into the system (the plane $z=z_{0}$ ) from its surroundings.

Once the fluctuations entered the system, the role of nonlinearities (second term in the right-hand side of eqs. [3] and [4]) is to redistribute them in $\boldsymbol{k}$-space. When the migration of this fluctuations in Fourier space statistically transfers invariants along a preferred direction in $k=|\boldsymbol{k}|$, the nonlinearities are said to be driving a cascade process. Ideal invariants are global quantities (integrated over the volume of the system) which are constants of motions of the ideal (nondissipative) set of equations. The purely two-dimensional and ideal MHD equations $\left(\partial_{z}=0\right.$ and $\eta=0=v_{1}$ in eqs. [3]-[4]) yield three global invariants: the energy $W$, the mean-square vector potential $A$ and the cross-helicity $H$

$$
\begin{gathered}
W=W_{U}+W_{B}=\frac{1}{2 D^{2}} \int d^{2} x\left\langle u^{2}+b^{2}\right\rangle \\
A=\frac{1}{2 D^{4}} \int d^{2} x\left\langle a^{2}\right\rangle, \\
H=\frac{1}{2 D^{2}} \int d^{2} x\langle\boldsymbol{u} \cdot \boldsymbol{b}\rangle,
\end{gathered}
$$

where the notation $\langle\ldots\rangle$ is used to indicate a statistical average over an infinite set of independent realisations of the dynamic evolution of the coronal loop under study.

When external forcing $\left(\partial_{z} \neq 0\right)$ and dissipation are allowed, the nonlinear terms produce a cascade of energy toward the large wavenumbers (direct cascade), while $A$ undergoes an inverse cascade and accumulates at the lowest wavenumbers (Fyfe et al. 1977). However, it has been shown (Gómez \& Ferro Fontán 1988) that this inverse cascade does not have observable consequences during the typical lifetime of a coronal loop. On the other hand, the direct energy cascade is responsible for the enhancement of Joule dissipation of the large-scale magnetic stresses driven by subphotospheric convection. Hereafter we shall concentrate on the study of this energy cascade and the resulting energy spectral function.

Under the assumption that this two-dimensional turbulence is isotropic (strictly valid for modes such that $k \gg 2 \pi / D$ ), any average over the turbulent ensemble will only depend upon $k=|\boldsymbol{k}|$. In this case, we can define global invariants per unit wavenumber

$$
\begin{gathered}
W(k)=\frac{D^{2} k}{4 \pi}\left\langle\left|\boldsymbol{u}_{k}\right|^{2}+\left|\boldsymbol{b}_{k}\right|^{2}\right\rangle, \quad W=\int d k W(k), \\
H(k)=\frac{D^{2} k}{8 \pi}\left\langle\boldsymbol{u}_{k} \cdot \boldsymbol{b}_{k}^{*}+\boldsymbol{u}_{k}^{*} \cdot \boldsymbol{b}_{k}\right\rangle, \quad H=\int d k H(k),
\end{gathered}
$$

For the sake of computing the brackets, we assume that the averaged variables are independent of $z$ (homogeneous turbulence) and that an ergodic hypothesis holds, thus allowing us to replace $\langle\ldots\rangle$ by a spatial average along $z$. Therefore, from equations (3) and (4) and using definitions (9)-(10), the evolution equation of the turbulent energy spectral function $W(k)$ results

$\partial_{t} W(k)+2 k^{2}\left[\eta W_{B}(k)+v_{1} W_{U}(k)\right]=v_{\mathrm{A}} \frac{H_{L}(k)-H_{0}(k)}{L}+T(k)$, where

$$
\begin{aligned}
T(k)=i\left(\frac{D}{2 \pi}\right)^{4} k & \int d^{2} k_{1} d^{2} k_{2} \delta\left(\boldsymbol{k}-\boldsymbol{k}_{1}-\boldsymbol{k}_{2}\right) \\
& \times\left\langle\boldsymbol{b}_{\boldsymbol{k}}^{*} \cdot \boldsymbol{k} \times\left(\boldsymbol{u}_{\boldsymbol{k}_{1}} \times \boldsymbol{b}_{\boldsymbol{k}_{2}}\right)+\boldsymbol{u}_{\boldsymbol{k}}^{*} \cdot\left(\boldsymbol{k}_{1} \times \boldsymbol{b}_{\boldsymbol{k}_{1}}\right) \times \boldsymbol{b}_{\boldsymbol{k}_{2}}\right\rangle
\end{aligned}
$$

represents the mean rate of excitation of modes $|\boldsymbol{k}|=k$ due to its interaction with the other ones. The global effect of these interactions is the aforementioned energy cascade from small to large wavenumbers. The second and third terms on the left-hand side in equation (11), correspond to Joule and viscous dissipation, respectively. The first term on the right-hand side expresses the work per unit time done by photospheric motions on the coronal magnetic field. The ergodic hypothesis establishes that this energy input is homogeneously distributed along the loop. This energy input rate is in fact a positive definite term, as has been shown by Chouduri 1986 (see also next section).

Equation (11) is expected to have stationary regimes among its solutions, expressing the balance at any wavenumber $k=|k|$ between the external energy input and the energy cascade toward large wavenumbers. At sufficiently high wavenumbers, the dissipative terms will eventually become large enough to quench the cascade and transform this energy into heat. In the next section we are going to extend the phenomenological model of Canuto et al. 1987 (developed for threedimensional hydrodynamic turbulence) to the description of two-dimensional MHD turbulence. This kind of models basically follow the same ideas that led Kolmogorov (1941) to discover his universal energy power spectrum. However, let us emphasize that Kolmogorov spectrum is valid in the energy inertial range of Fourier space (i.e., in the spectral region bridging the gap between the externally forced and the dissipative regions), while the present model is expected to describe the energy spectrum in the externally forced region. Depending on the spectral shape of the external force, the system may or may not develop an energy inertial range at large wavenumbers.

\section{A SIMPLE MODEL FOR LARGE-SCALE TURBULENCE}

The heuristic model for a fully turbulent fluid proposed by Canuto et al. (1987), essentially describes a global balance between energy rates in the Fourier range $\left(k_{0}, k\right)$, where $k_{0}$ is the lowest wavenumber of the system. The cascade rate $T(k)$ continually drains energy from this spectral region [transferring it to the range $(k, \infty)$ ], thus acting as a turbulent or eddy viscosity. Therefore

$$
\epsilon(k)=\left(v+v_{t}\right) \Omega(k),
$$

where

$$
\epsilon(k)=\int_{k_{0}}^{k} d k f(k),
$$

is the external energy input in the range $\left(k_{0}, k\right)$ and

$$
\Omega(k)=\int_{k_{0}}^{k} d k k^{2} W(k),
$$

is the corresponding enstrophy. The parameter $v$ is the molecular viscosity and

$$
v_{t}(k)=\int_{k}^{\infty} d k \frac{W(k)}{n(k)},
$$

is the total eddy viscosity at $|\boldsymbol{k}|=k$ excited by modes with wavenumbers larger than $k$, while $n(k)$ is the rate of transfer of 
energy induced by the nonlinear interactions. By definition, the correlation rate $n(k)$ is proportional to the rate at which energy cascades from $k$ to wavenumbers larger than $k$. Therefore

$$
\gamma n(k)=v_{t}(k) k^{2} \text {. }
$$

The new parameter $\gamma$ is a function of the correlation rate at the lowest wavenumber of the system $\left(k_{0}\right)$ through the relation

$$
\gamma \frac{n\left(k_{0}\right)}{k_{0}^{2}}=\int_{k_{0}}^{\infty} d k \frac{W(k)}{n(k)} \text {. }
$$

The stationary energy spectrum $W(k)$ and the correlation rate $n(k)$ can be consistently derived from the set of integral equations (13)-(18). Taking derivatives with respect to $k$, these equations can be transformed into a set of ordinary differential equations (ODEs) coupled with an integral condition for the parameter $\gamma$ (see $\S 4)$.

We are interested in transforming the energy balance for MHD turbulence shown in equation (11) (in the limit $\partial_{t}=0$ ) to an equation equivalent to equation (13). Therefore, we integrate equation $(11)$ in $\left(k_{0}, k\right)$

$$
\frac{1}{\tau_{\mathrm{A}}} \int_{k_{0}}^{k} d k \Delta H=2 \eta \int_{k_{0}}^{k} d k k^{2} W_{B}+2 v_{1} \int_{k_{0}}^{k} d k k^{2} W_{U}-\int_{k_{0}}^{k} d k T
$$

where $\Delta H=H_{L}-H_{0}$ and $\tau_{\mathrm{A}}=L / v_{\mathrm{A}}$ is the Alfvén transit time along the loop. We define

$$
\begin{gathered}
\Omega(k)=\int_{k_{0}}^{k} d k k^{2} W(k), \\
v_{t}(k) \Omega(k)=-\int_{k_{0}}^{k} d k T(k), \\
f(k)=\frac{1}{\tau_{\mathrm{A}}} \Delta H(k) .
\end{gathered}
$$

Because in the corona is $|\boldsymbol{u}| \ll|\boldsymbol{b}|$, we neglect $W_{U}$ with respect to $W_{B}$ and asume that the energy dissipation is essentially ohmic $\left(\eta W_{B} \gg v_{1} W_{U}\right.$, see Gómez \& Ferro Fontán 1988). Under these definitions and assumptions, whose validity can be checked a posteriori, equation (19) for MHD turbulence in the solar corona is similar to the equations of the heuristic model of Canuto et al. (1987).

The crucial part of this model is the representation of the nonlinear interactions as an eddy viscosity (as shown in eqs. [16] and [21]), also the proportionality expressed in equation (17), that allows the closure of the set of equations. This closure relationship can also be derived from dimensional arguments. Let $u_{k}$ be the velocity amplitude at wavenumber $k$. Therefore, the cascade rate $n(k)$ should be $n(k) \propto\left(k u_{k}\right)^{-1}$ and the eddy viscosity $v_{t}(k) \propto u_{k} / k$. Removing $u_{k}$ in these relationships and defining the proportionality constant $\gamma$, automatically leads to equation (17). It is straightforward to see that this derivation also holds for the MHD case. Equation (12) shows that the efficiency for nonlinear transfer of magnetic energy $\left(W \approx\left\langle b^{2}\right\rangle\right)$, is directly related to the Fourier amplitude of the velocity field $\left(T \approx\left\langle u b^{2}\right\rangle\right)$. We note here that even though we assumed $|\boldsymbol{u}| \ll|\boldsymbol{b}|$, the role of the velocity field is still essential in controlling the cascade rate of magnetic energy.

After considering some simplifying assumptions for the stressing mechanism, we derive an expression for the forcing term $f(k)$. Suppose, for example, that the subphotospheric kinetic energy spectrum can be represented by a power law $W_{\text {ph }}(k) \approx k^{-\alpha}$. We impose a cutoff of this spectrum at the lowest wavenumber $k_{0}$ (which has been chosen to correspond to a length scale of about twice the size of granules) in the following way:

$$
W_{\mathrm{ph}}(k)=C q^{-\alpha}\left(\frac{q^{\beta}}{1+q^{\beta}}\right), \quad q=\frac{k-k_{0}}{k_{0}},
$$

where, for example, the power $\alpha$ is $5 / 3$ in a Kolmogorov spectrum, and $\beta \gg 1$ is an ad hoc parameter defining the steepness of the cutoff, while the constant $C$ equals

$$
C=W_{\mathrm{ph}} \frac{\beta}{\alpha}\left(\frac{\beta}{\alpha}-1\right)^{\alpha / \beta-1} .
$$

The constant $W_{\mathrm{ph}}$ is the maximum value of the subphotospheric spectrum, attained at $k \approx 2 k_{0}$, and which we associate with the granular convection cells.

With respect to the stressing of magnetic field lines, we assume that the uncorrelated footpoint displacements $\boldsymbol{u}_{0} t$ and $u_{L} t$ applied to the respective ends of a given field line during a time interval $t$, generate a magnetic field component $\boldsymbol{b} \simeq$ $v_{\mathrm{A}}(\Delta u t / L)=\Delta u\left(t / \tau_{\mathrm{A}}\right)$, as we schematically show in Figure 1 . If we associate Fourier modes of modulus $k=|\boldsymbol{k}|$, with eddies of size $k^{-1}$, characteristic velocity $\boldsymbol{u}_{k}$ and correlation time $\tau_{k}$, we can estimate that a relative displacement $\Delta \boldsymbol{u}_{\boldsymbol{k}} \tau_{\boldsymbol{k}}$ between footpoints during the correlation time $\tau_{k}$ (lifetime of the eddy), causes a coronal magnetic field component $\boldsymbol{b}_{\boldsymbol{k}}=\Delta \boldsymbol{u}_{\boldsymbol{k}}\left(\tau_{\boldsymbol{k}} / \tau_{\mathrm{A}}\right)$. Therefore, from equations (10) and (22) we can derive the following expression for the external driving force:

$$
f(k)=\frac{\tau_{k}}{\tau_{\mathrm{A}}^{2}} \frac{D^{2} k}{4 \pi}\left\langle\Delta \boldsymbol{u}_{k} \Delta \boldsymbol{u}_{k}^{*}\right\rangle=\frac{2 \tau_{k}}{\tau_{\mathrm{A}}^{2}} W_{\mathrm{ph}}(k),
$$

where $W_{\text {ph }}(k)=\left(D^{2} k / 4 \pi\right)\left\langle\left|\boldsymbol{u}_{k}\right|^{2}\right\rangle$. To obtain equation (25) we used the fact that $\boldsymbol{u}_{0}$ and $\boldsymbol{u}_{L}$ are uncorrelated (thus $\left\langle\left|\Delta \boldsymbol{u}_{k}\right|^{2}\right\rangle=$ $\left.2\left\langle\left|u_{k}\right|^{2}\right\rangle\right)$. According to Kolmogorov, the eddy correlation time is

$$
\tau_{k} \simeq\left(k \tilde{u}_{k}\right)^{-1} \simeq k^{-3 / 2} W_{\mathrm{ph}}^{-1 / 2},
$$

since $W_{\mathrm{ph}}(k) \simeq\left(\tilde{u}_{k}^{2} / k\right)$. The tilded variable $\tilde{u}_{k}$ is a real and positive number with velocity dimensions, defined to measure the spectral intensity at wavenumber $k$, and should not be confused with the random variable $\boldsymbol{u}_{k}$. Therefore, taking these scaling arguments into account, we define the forcing term $f(k)$ as

$$
f(k)=\frac{W_{\mathrm{ph}}^{1 / 2}(k)}{k^{3 / 2} \tau_{\mathrm{A}}^{2}} .
$$

Expression (26) is the forcing term that we employ to solve equations (13)-(18). We would like to point out that the relationship $\boldsymbol{b}_{\boldsymbol{k}}=\Delta \boldsymbol{u}_{\boldsymbol{k}}\left(\tau_{k} / \tau_{\mathrm{A}}\right)$ reasonably holds for $\tau_{\boldsymbol{k}}>\tau_{\mathrm{A}}$. For turnover times shorter than the Alfvén time $\left(\tau_{k}<\tau_{\mathrm{A}}\right)$, we could consider instead that Alfvén waves are being generated (Heyvaerts \& Priest 1984) and that $\boldsymbol{b}_{\boldsymbol{k}}= \pm \boldsymbol{u}_{\boldsymbol{k}}$ should be used. However, we have found that at least for a subphotospheric kinetic energy spectrum $W_{\mathrm{ph}}(k) \approx k^{-5 / 3}$, the excitation time $\tau_{k}$ decreases very slowly with $k\left(\tau_{k} \approx k^{-2 / 3}\right)$ and becomes comparable to $\tau_{\mathrm{A}}$ at wavenumbers $k \approx \tau_{\mathrm{A}}^{-3 / 2} W_{\mathrm{ph}}^{-3 / 4} k_{0}^{-5 / 4} \simeq 200 k_{0}$. Hence, we find that our assumption $b_{k}=\Delta \boldsymbol{u}_{k}\left(\tau_{k} / \tau_{\mathrm{A}}\right)$ for the externally driven fields, is correct for the energy containing spectral region. However, it is well-known that at large wavenumbers the system tends to an equipartition between kinetic 
and magnetic energy, slowly approaching this state like $W_{B}$ $-W_{U} \approx k^{-2}$ (Pouquet, Frisch, \& Leorat 1976; see also Biskamp \& Welter 1989). This relative increase of kinetic energy at large wavenumbers may cause viscous effects to be important or even dominant, as discussed by Hollweg (1986).

We remark that the stressing mechanism presented above is of the same nature of the ones proposed by Parker (1983b) or Sturrock \& Uchida (1981). The main difference is that we assume a broad-band spectrum of subphotospheric motions instead of one sharply peaked at the granules size. However, regardless of the spectral details of these models, the estimated energy input roughly matches the energy requirements for coronal active regions $\left(10^{5}-10^{7}\right.$ ergs $\left.\mathrm{cm}^{-2} \mathrm{~s}^{-1}\right)$. The energy flowing into the loop per unit area and time is

$$
F=2 \rho L \int_{k_{0}}^{\infty} d k f(k) \approx 2 \rho L\left[2 k_{0} f\left(2 k_{0}\right)\right],
$$

because $f(k)$ is a piecewise power-law function, peaked at $k \approx 2 k_{0}$. Using equation (26) and $\left(2 k_{0}\right) W_{\mathrm{ph}} \approx \frac{1}{2} u_{\mathrm{ph}}^{2}$ (also $\left.2 k_{0} \approx\left[1 / u_{\mathrm{ph}} \tau_{\mathrm{ph}}\right]\right)$, we obtain

$$
F=\frac{B_{0}^{2}}{4 \pi L} u_{\mathrm{ph}}^{2} \tau_{\mathrm{ph}} \approx 8 \times 10^{6} B_{2} L_{9}^{-1} \text { ergs } \mathrm{cm}^{-2} \mathrm{~s}^{-1},
$$

where $u_{\mathrm{ph}}$ and $\tau_{\mathrm{ph}}$ are the typical velocity and turnover time for granules' motions, $B_{2}$ is in $10^{2} \mathrm{G}$ units and $L_{9}$ in $10^{9} \mathrm{~cm}$ units.

In the next section we will transform equations (13)-(18) into a set of ODEs and show the results from their numerical integration.

\section{NUMERICAL RESULTS}

The derivative with respect to $k$ of equation (13) gives

$$
f(k)+\frac{W(k)}{n(k)} \Omega(k)=\left(\eta+v_{t}\right) k^{2} W(k),
$$

representing a local energy balance in wavenumber space. The left-hand side displays the energy input rates to modes with $|\boldsymbol{k}|=k$. The first term corresponds to the external input, while the second term is the energy coming from smaller wavenumbers. The right-hand side represents the effective energy loss due to both molecular and eddy dissipation. Using (17) we can replace $v_{t}(k)$ in (29) to obtain

$$
\Omega(k)=-\frac{n f}{W}+n k^{2}\left(\eta+\gamma \frac{n}{k^{2}}\right) .
$$

Taking the derivatives of equations (30) and (17), we finally get

$$
\begin{gathered}
k^{2} W=-\partial_{k}\left(\frac{n f}{W}-\eta n k^{2}-\gamma n^{2}\right), \\
\gamma \partial_{k}\left(\frac{n}{k^{2}}\right)=-\frac{W}{n},
\end{gathered}
$$

which is a set of first-order ODEs for $n$ and $W$. We are going to solve these equations for all $k$ larger than $k=k_{0}$, using as "initial conditions" $W\left(k_{0}\right)=0$ and $n\left(k_{0}\right)=n_{0} \simeq \tau_{k_{0}}^{-1}$, where $\tau_{k_{0}}$ is the typical turnover time for subphotospheric eddies. These initial conditions are not chosen arbitrarily, but arise from asymptotic requirements at $k \simeq k_{0}$ for the original equations (17) and (29). Also, the solution of equations (31)-(32) must satisfy the integral condition (18), which can be considered as an equation for the unknown $\gamma$. Actually, equations (31)-(32) and (18) were consistently solved following an iterative procedure. We found a degree of numerical instability for certain values of $\gamma$, an effect also reported by Canuto et al. (1987).

In Figure $2 a$ we show the magnetic energy spectrum $W(k)$ that we obtained. We have found that for large values of $k$, the turbulent magnetic energy spectrum asymptotically approaches a Kolmogorov slope $-5 / 3$. For comparison, Figure $2 a$ also shows the spectrum for subphotospheric kinetic energy $W_{\mathrm{ph}}(k)$. At wavenumbers around the peak of $W_{\mathrm{ph}}(k)$ and based on the characteristics of the excitation mechanism we proposed (see $\S 3$ ), we expect the coronal velocity field to be comparable to the subphotospheric field. This assertion does not necessarily hold in the cascading region $\left(k>2 k_{0}\right)$, since the total energy cascade might contribute to raise the amplitude of velocity fluctuations. The coronal velocity amplitude in this region can be estimated as $\tilde{\boldsymbol{u}}_{k} \approx n(k) / k$ and, therefore, the kinetic energy spectrum becomes

$$
W_{U} \simeq \frac{\tilde{u}_{k}^{2}}{k} \propto \frac{n^{2}(k)}{k^{3}}, \quad k \gg k_{0} .
$$

We chose the proportionality constant implicit in equation (33) in such a way that the coronal kinetic energy per unit mass $W_{\mathrm{U}}(k)$ asymptotically approaches $W_{\text {ph }}(k)$ toward the lowwavenumber region, as can be seen in Figure $2 a$. At large wavenumbers, we can see that the coronal kinetic energy per unit mass also approaches a power-law spectrum $k^{-5 / 3}$, however with an amplitude somewhat larger than the photospheric kinetic energy. The forcing term $f(k)$ that we used is shown in Figure $2 b$, displaying a slope $-7 / 3$ at high wavenumbers.

We have found that equations (31)-(32) asymptotically approach (at large wavenumbers) a solution that can be described analytically. This asymptotic solution corresponds to the $-5 / 3$ slope, for both the magnetic and kinetic energy spectrum. It is characterized by a constant value for the term

$$
\frac{n(k) f(k)}{W(k)}=c
$$

which we numerically found to be $c / n_{0}^{2} \simeq 81.2$. With the aid of equation (34), equations (31)-(32) can be readily integrated. We obtain

$$
\begin{gathered}
W(k)=\frac{3}{4 \gamma c^{2}} k^{3} f^{2}(k)=\frac{3}{4 \gamma c^{2} \tau_{\mathrm{A}}^{4}} W_{\mathrm{ph}}(k), \\
n(k)=\frac{3}{4 \gamma c} k^{3} f(k), \\
\frac{W_{U}(k)}{W(k)}=\frac{3}{4 \gamma} \simeq 0.042 .
\end{gathered}
$$

In Figure 3 we show how this asymptotic state is reached. Figure $3 a$ displays the ratio between coronal and photospheric kinetic energy densities per unit mass. We see that this fraction reaches a constant asymptotic value of $W_{U} / W_{\mathrm{ph}} \approx 1.67$. Figure $3 b$ shows the ratio between coronal kinetic energy and magnetic energy. We can see that even though this ratio monotonically increases with $k$, it approaches an asymptotic value $W_{U} / W \approx 0.042$. This result is consistent with our assumption $|\boldsymbol{u}| \ll|\boldsymbol{b}|$. We found that the approximation of our numerical solution to this asymptotic state, critically depends on the value of $\gamma$. Therefore, we iterated our numerical code until $\gamma$ was determined with a precision of better than $10^{-7}$. 

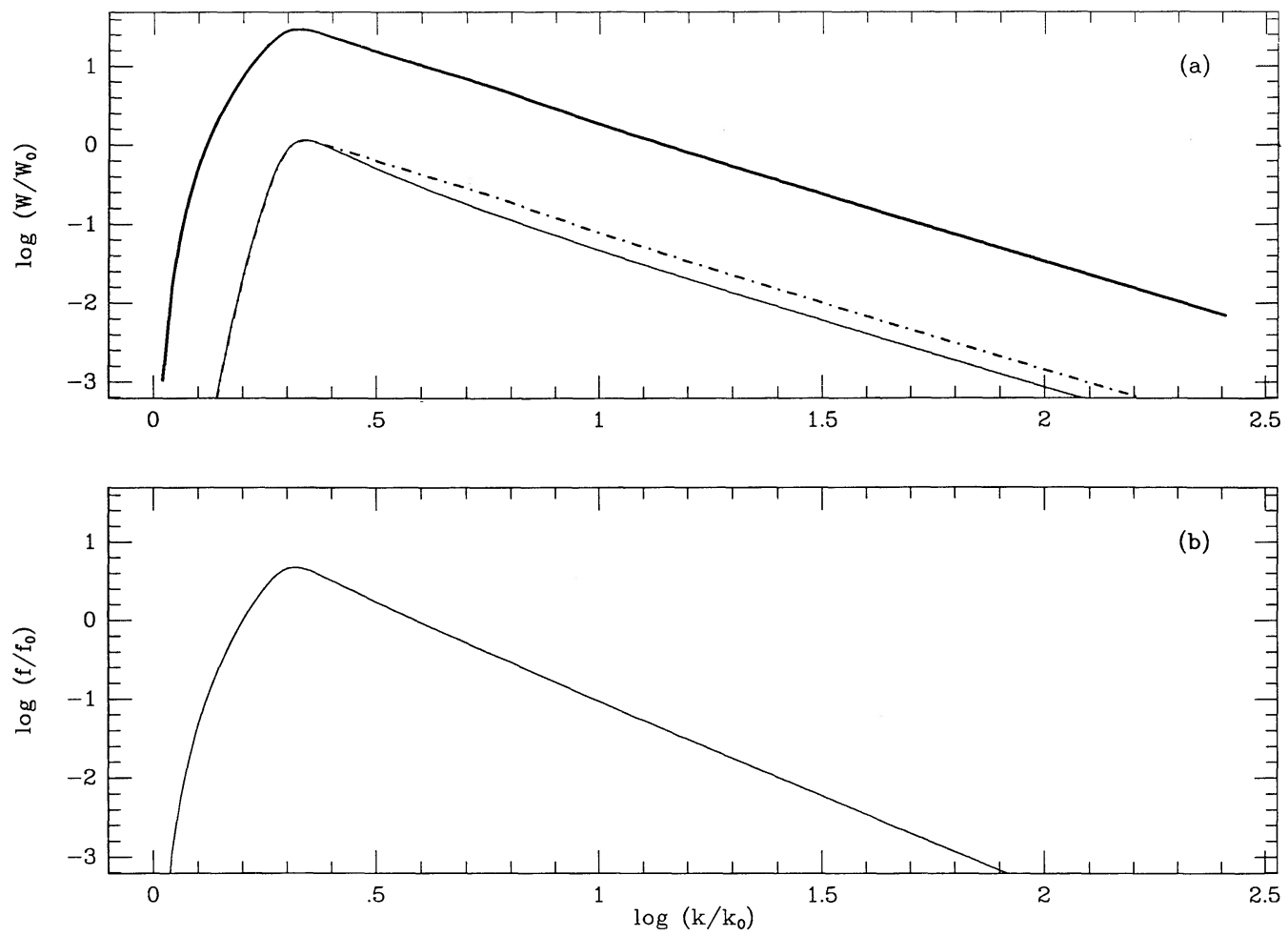

Fig. 2.-(a) Stationary power spectra for magnetic energy (thick line), coronal kinetic energy (dot-dashed line) and subphotospheric kinetic energy (thin line). These are energy densities per unit mass and all of them asymptotically approach a slope $-5 / 3$ at high wavenumbers. (b) Power spectrum for the external force.
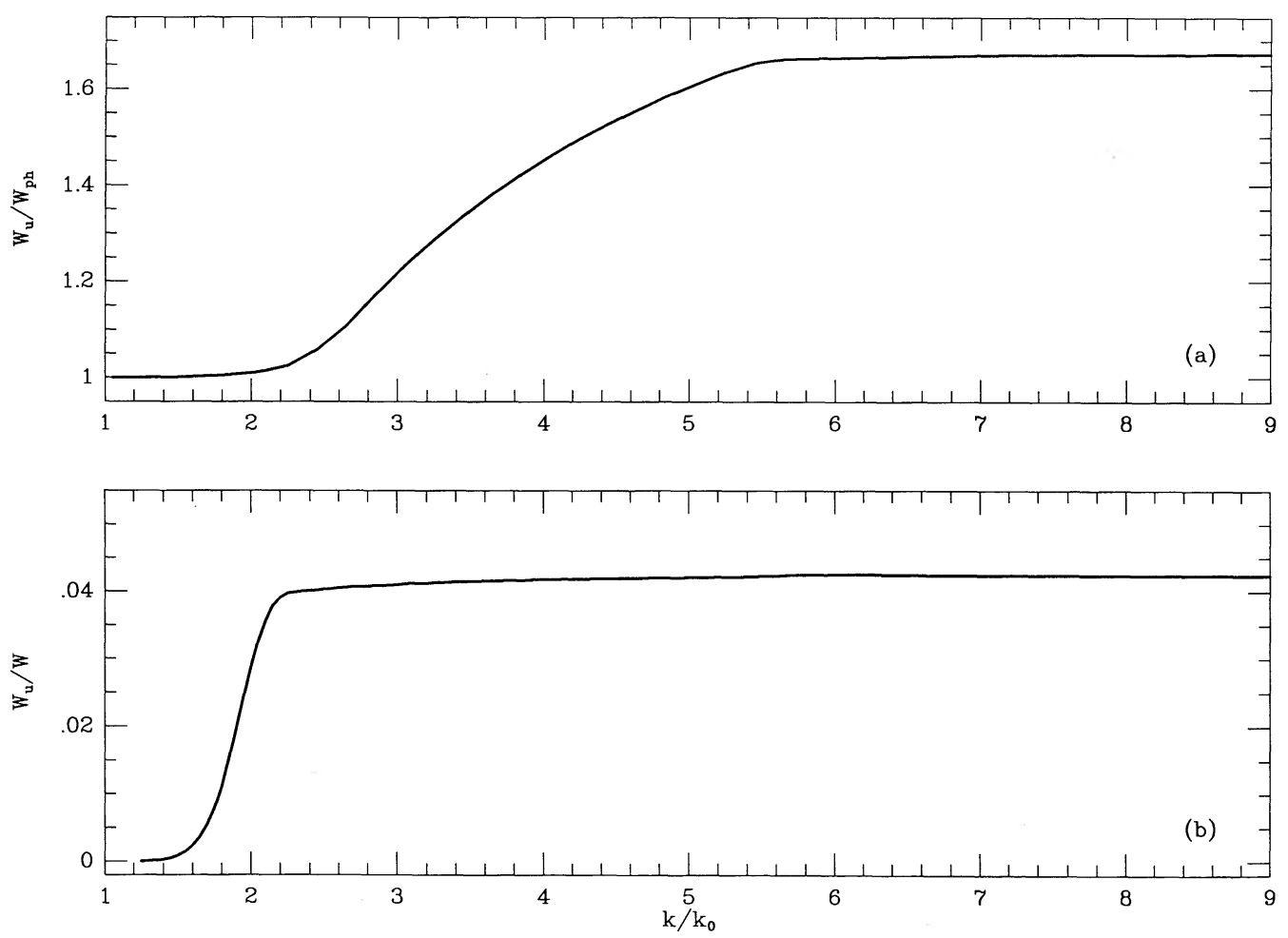

FIG. 3.-(a) Ratio of coronal to subphotospheric kinetic energy density per unit mass. (b) Ratio of kinetic to magnetic energy density per unit mass in the corona 


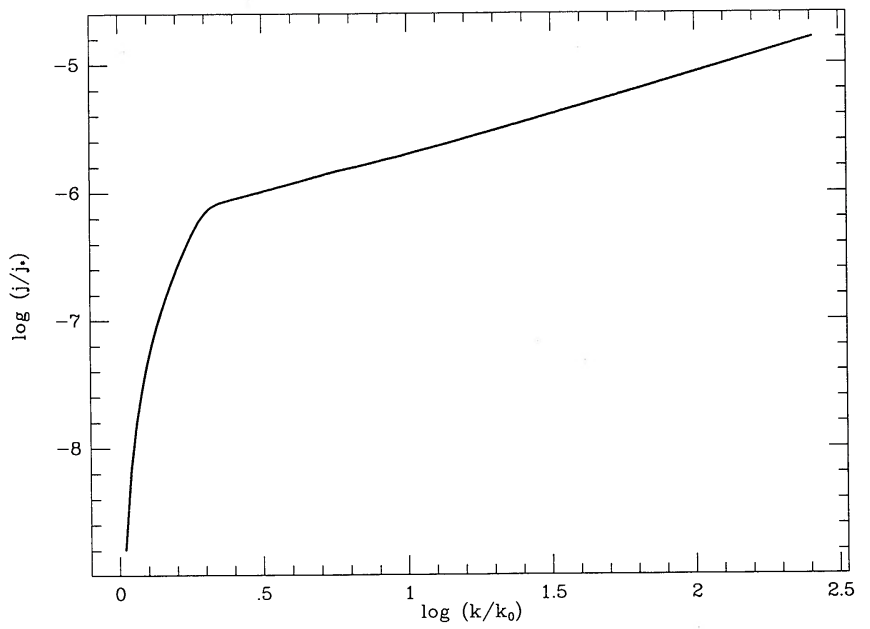

FIG. 4.-Stationary power spectrum of the turbulent electric current density in units of the threshold value for ion-acoustic instabilities $\left(j_{*}\right)$. The $x$-axis is $\log \left(k / k_{0}\right)$, and the $y$-axis is $\log \left(j / j_{*}\right)$.

For the numerical integration we used dimensionless variables with $k_{0}=4.10^{-8} \mathrm{~cm}^{-1}$ and $n_{0}=1.10^{-3} \mathrm{~s}^{-1}$ as typical coronal values. The Joule resistivity that we used is $\eta=6.10^{4}$ $\mathrm{cm}^{2} \mathrm{~s}^{-1}$. The maximum of the subphotospheric energy spectral function is $W_{\mathrm{ph}}=6.10^{16} \mathrm{~cm}^{3} \mathrm{~s}^{-2}$, based on observational data of granular motions $\left(u_{\mathrm{ph}} \approx 1 \mathrm{~km} \mathrm{~s}^{-1}, \tau_{\mathrm{ph}} \approx 1000 \mathrm{~s}\right)$. The Alfvén time $\tau_{\mathrm{A}}$ is around $20 \mathrm{~s}$ and the loop length is $L \approx 3.10^{9} \mathrm{~cm}$. For the cutoff coefficient $\beta$ we chose $\beta=10$, but we found that our results do not depend critically upon this parameter.

The enstrophy $\Omega(k)$, for this MHD case, is the spectral function of the squared electric current density flowing through the plasma. In Figure 4 we show the spectral behavior of this turbulent current $j(k)=\left(k^{3} W\right)^{1 / 2}$ in units of $j_{*}=2.15$ $e n\left(T_{e} / m_{i}\right)^{1 / 2}$, which is the threshold value for the excitation of ion-acoustic turbulence. As shown in Figure 4, $j(k)$ remains much smaller than $j_{*}$ throughout the displayed range of $k$, increasing very slowly as $k^{2 / 3}$. Therefore, it is very unlikely that the stochastic current distribution associated with MHD turbulence can develop ion-acoustic turbulence. One of the main effects of ion-acoustic turbulence is to raise the resistivity in about four orders of magnitude (for typical coronal conditions), with the corresponding increase in the Joule dissipation rate. This topic will be further discussed in the next section.

\section{DISCUSSION}

The aim of the present work has been to calculate the stationary energy spectrum of MHD turbulence in coronal loops driven by subphotospheric convective motions. The importance of the development of MHD turbulence relies on the enhancement of Joule heating due to the energy cascade towards the microscopic wavenumbers. From equations (31)(32) we can derive the energy power spectrum of coronal MHD turbulence for a given energy spectrum of subphotospheric turbulence. Regretfully, the detailed features of the spectral distribution of subphotospheric kinetic energy are still unknown. Just as an illustrative example, we analyzed the effect of a Kolmogorov spectrum for the convective region $\left(W_{\mathrm{ph}} \approx k^{-5 / 3}\right)$. Our numerical results indicate that both magnetic and kinetic energy in the corona, asymptotically tend to a $k^{-5 / 3}$ power-law at large wavenumbers. Nonetheless, we find that the fraction of kinetic to magnetic energy remains as small as $4 \%$, which is consistent with our hypothesis of a magnetically dominated regime.

As stressed in the introduction, a power-law spectrum $W(k) \approx k^{-5 / 3}$ is also expected to develop for the case of an external driver sharply peaked in Fourier space. In the context of the coronal heating problem, it corresponds to consider only the driving action of the granules structures (Gómez \& Ferro Fontán 1988). In order to test to what degree our cascading range $\left(k>2 k_{0}\right)$ can be considered an energy inertial region, we computed the ratio $q(k)=f(k) /\left[v_{t} k^{2} W(k)\right]$, which measures the relative importance of the external input rate compared to the eddy damping rate (see eq. [29]). We found that this ratio remains quite close to $q \simeq 1$ in the range $k / k_{0} \simeq 1-3$ and then falls off like $k^{-4 / 3}$, reaching a value of $q \simeq 0.01$ at $k / k_{0} \simeq 60$. This result shows that even though the driving mechanism is broad-band, its energy input rate becomes gradually negligible compared to the energy-cascading rate, giving birth to an energy inertial range. We remind at this point that, for $k / k_{0} \geq$ 200 (when $\tau_{k} \approx \tau_{\mathrm{A}}$ ), the expression for the external force (eq. [26]) should be changed to allow for the energy input carried by traveling Alfvén waves. However, we speculate that at this very large wavenumber range the relative importance of the external energy input is so small that any modification of this term will not seriously affect the cascading process.

We find that molecular dissipation begins to be relevant $\left[\eta \approx v_{t}(k)\right]$ for a wavenumber $k_{\eta}$ given by

$$
\frac{k_{\eta}}{k_{0}}=4.10^{5},
$$

which corresponds to scales much smaller than the electron mean free path $\left(\lambda_{e} \approx 5.10^{4} \mathrm{~m}\right)$ and much larger than the electron giroradius $\left(r_{e} \approx 0.2 \mathrm{~cm}\right)$.

Recent numerical simulations performed by Biskamp \& Welter (1989) show the development of a strongly intermittent turbulent state, where the electric current distribution reveals the presence of Sweet-Parker current sheets. These dissipative structures evolve stretching and folding until the tearing instability disrupts them. This behavior leads to a dense distribution of current microsheets, remarkably resembling Parker's scenario of spontaneous current sheet formation (Parker 1981a, b, 1983a, b). Biskamp \& Welter (1989) claim that these current sheets have a typical thickness $k_{\eta}^{-1}$, their width and mean separation being of the order of the Taylor microscale $\lambda=\left(W_{\eta} / \epsilon\right)^{1 / 2}$. For the coronal turbulence, the Taylor microscale is $\lambda \approx\left(\tau_{\mathrm{ph}} \eta\right)^{1 / 2} \approx 70 \mathrm{~m}$.

The development of ion-acoustic turbulence in current sheets has usually been invoked as a means of enhancing Joule dissipation (for instance, Rosner et al. 1978). Hence, we decided to study the feasibility of the excitation of ion-acoustic turbulence. Whenever this turbulence develops, an important increase in the resistivity coefficient results $\left(\eta_{\mathrm{IA}} \approx 10^{4} \eta\right.$ in the solar corona). Nevertheless, note that as long as a stationary spectrum of MHD turbulence is present, the end result of an enhanced resistivity will not be an increase of the heating rate, but a strong reduction of the dissipation wavenumber $k_{\eta}$.

For the development of ion-acoustic turbulence, the electric current density must exceed the critical value $j_{*}=2.15$ en $\left(T_{e} / m_{i}\right)^{1 / 2}$ (see Kaplan \& Tsytovitch 1973 or Bichenkov \& Silin 1982). We calculated the turbulent spectral function for the current density to find out whether there is a region in the wavenumber space where this threshold value is surpassed. 
However, in Figure 4 we see that $j(k)$ is quite low for any value of $k$, increasing only as $k^{2 / 3}$. Therefore, we conclude that within the framework of the present theory, it is unlikely to expect that ion-acoustic turbulence is being triggered by a stochastic distribution of electric currents.

In conclusion, we have derived the spectrum for MHD turbulence in coronal loops, generated by a Kolmogorov spectral distribution of the subphotospheric hydrodynamic turbulence. We find that at wavenumbers larger than the inverse of the granulation length scale, a magnetically dominated stationary energy spectrum $W(k) \approx k^{-5 / 3}$ takes place. The turbulent MHD energy cascades toward very microscopic scales until it is quenched by Joule dissipation.
The authors would like to thank Marcos Machado, Piet Martens, George Roumeliotis and Aad van Ballegooijen, for numerous stimulating discussions that substantially contributed to improve an earlier version of this manuscript. Useful suggestions from an anonymous referee are also acknowledged.

One of us (D. G.) also acknowledges the hospitality of the Harvard-Smithsonian Center for Astrophysics, where the writing of this paper has been performed.

This work was partially supported by the Consejo Nacional de Investigaciones Científicas y Técnicas (CONICET, Argentina) under grant $9069 / 03$ and by the University of Hawaii under grants NAGW864 from NASA and ATM9106052 from NSF.

\section{REFERENCES}

Batchelor, G. K. 1953, The Theory of Homogeneous Turbulence (Cambridge: Cambridge Univ. Press)

Bichenkov, V., \& Silin, V. P. 1982, J. Exper. Theoret. Phys., 55, 1086

Biskamp, D., \& Welter, H. 1989, Phys. Fluids, B1, 1964

Braginskii, S. I. 1965, Rev. Plasma Phys., 1, 205

Canuto, V. M., Cabot, W., Hartke, G. J., \& Battaglia, A. 1985, Ap\&SS, 116, 367

Canuto, V. M., \& Goldman, I. 1985, Phys. Rev. Lett., 54, 430

Canuto, V. M., Goldman, I., \& Chasnov, J. 1987, Phys. Fluids, 30, 3391

Canuto, V. M., Goldman, I., \& Hubickyj, O. 1984, ApJ, 280, L55

Chou, D. Y., LaBonte, B. J., Braun, D. C., \& Duvall, T. L. 1991, ApJ, 372, 314

Chouduri, A. R. 1986, in Coronal and Prominence Plasmas, ed. A. I. Poland (NASA CP 2442), 451

Fyfe, D., Joyce, G., \& Montgomery, D. 1977, J. Plasma Phys., 17, 317

Fyfe, D., Montgomery, D., \& Joyce, G. 1977, J. Plasma Phys., 17, 369

Gómez, D. 1990, Fund. Cosmic Phys., 14, 131

Gómez, D., \& Ferro Fontán, C. 1988, Solar Phys., 116, 33

Hasegawa, A. 1985, Adv. Phys., 35, 1

Heisemberg, W. 1948, Proc. Roy. Soc. London A, 195, 402

Heyvaerts, J., \& Priest, E. R. 1984, A\&A, 137, 63

Hollweg, J. 1983, in Solar Wind V, ed. M. Neugebauer (NASA CP 2280), 5

-.1986, ApJ, 306, 730
Kaplan, S. A., \& Tsytovitch, V. N. 1973, Plasma Astrophysics (New York: Pergamon), 134

Kolmogorov, A. N. 1941, C. R. Acad. Sci., USSR, 30, 301

Kraichnan, R. H. 1965, Phys. Fluids, 8, 575

Kuperus, M., Ionson, J. A., \& Spicer, D. A. 1981, ARA\&A, 19, 7

Narain, U., \& Ulmschneider, P. 1990, Space Sci. Rev., 54, 377

Parker, E. N. 1981a, ApJ, 244, 631 $.1981 \mathrm{~b}$, ApJ, 244, 644

1983a, ApJ, 264, 635

1983b, ApJ, 264, 642

Politano, H., Pouquet, A., \& Sulem, P. L. 1989, Phys. Fluids, B1, 2330

Pouquet, A., Frisch, U., \& Leorat, J. 1976, J. Fluid Mech., 77, 321

Rosner, R., Golub, L., Coppi, B., \& Vaiana, G. 1978, ApJ, 222, 317

Sturrock, P. A., \& Uchida, Y. 1981, ApJ, 246, 331

Tarbell, T. 1992, in Electromechanical Coupling in the Solar Atmosphere, ed. D. Spicer \& P. MacNeice (NASA CP), in press

Vaiana, G. S., \& Rosner, R. 1978, ARA\&A, 16, 393

Van Ballegooijen, A. A. 1986, ApJ, 311, 1001

Zahn, J. P. 1987, in Solar and Stellar Physics, ed. E. H. Schroter \& M. Schussler (Berlin: Springer), 55 